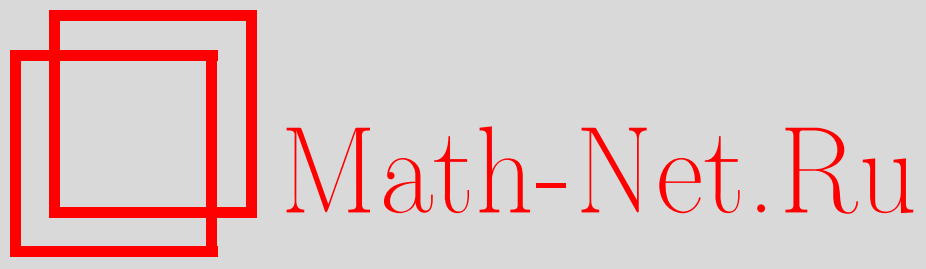

Г. М. Губреев, Структура модельных вольтерровых операторов, биортогональные разложения и интерполяция в регулярных пространствах де Бранжа, Функи. анализ и его прил., 2001, том 35, выпуск $2,74-78$

DOI: https://doi.org/10.4213/faa248

Использование Общероссийского математического портала MathNet.Ru подразумевает, что вы прочитали и согласны с пользовательским соглашением

http://www. mathnet.ru/rus/agreement

Параметры загрузки:

IP : 54.198 .67 .100

26 апреля 2023 г., 15:21:45

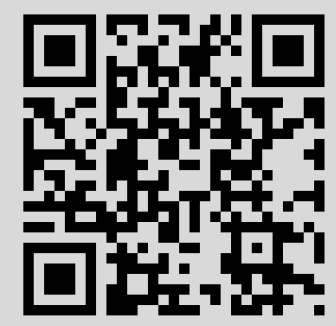


Модулярные формы и операторы Гекке. Наука, М., 1990; English transl.: Transl. Math. Monographs, Vol. 145, Amer. Math. Soc., Providence, R.I., 1995. 3. Ikeda T. On lifting of elliptic modular forms to Siegel cusp forms of degree $2 n$, Preprint, Kyoto University, 1999. 4. Langlands R. P. Euler products, Yale Mathematical Monographs, Vol. 1, Yale University Press, New Haven-London, 1971.

С.-Петербургское отделение Математического института им. В. А. Стеклова РАН 28 сентября 2000 г.

УДК 517.43

\title{
Структура модельных вольтерровых операторов, биортогональные разложения и интерполяция в регулярных пространствах де Бранжа*
}

\author{
(C) 2001. Г. М. ГувРеЕв
}

Заметка посвящена одному из возможных приложений результатов работы [1], полные доказательства которых приведены в [2]. Вместе с тем она написана так, что для ее понимания знакомство с работами $[1,2]$ не обязательно.

1. Регулярные пространства де Бранжа. Пусть целая функция $E$ удовлетворяет условию $|E(\bar{z})|<|E(z)|, \operatorname{Im} z>0$. Пространством де Бранжа $\mathscr{H}(E)$ (см. [3]) называется совокупность таких целых функций $f$, что: 1) $f(z) / E(z), f^{*}(z) / E(z)$ суть функции ограниченного вида и неположительного среднего типа в области $\operatorname{Im} z>0$; 2) $\|f\|_{\mathscr{H}(E)}^{2}:=\int_{R}|f(x)|^{2}|E(x)|^{-2} d x<\infty$.

Здесь (и далее) $f^{*}(z):=\overline{f(\bar{z})}$, а средний тип $h(F)$ аналитической в области $\operatorname{Im} z>0$ функции $F$ определяется равенством

$$
h(F):=\limsup _{y \rightarrow+\infty} y^{-1} \log |F(i y)| .
$$

Пространство $\mathscr{H}(E)$ является гильбертовым относительно скалярного произведения $\langle f, g\rangle$, согласованного с нормой $\|f\|_{\mathscr{H}(E)}^{2}$. Записав $E$ в виде $E(z)=$ $a(z)-i b(z)$, где $a, b-$ вещественные целые функции, рассмотрим ядро

$$
k(\lambda, z)=(b(\lambda) \overline{a(z)}-a(\lambda) \overline{b(z)}) / \pi(\lambda-\bar{z}),
$$

которое является воспроизводящим для $\mathscr{H}(E)[3]$, т. е.

$$
\langle f(\lambda), k(\lambda, z)\rangle=f(z), \quad f \in \mathscr{H}(E), z \in \mathbb{C} .
$$

Целая функция $S$ называется сопутствующей пространству $\mathscr{H}(E)$, если 1) $S(z) / E(z), S^{*}(z) / E(z)$ - функции ограниченного вида и неположительного среднего типа в $\operatorname{Im} z>0 ; 2) \int_{\mathbb{R}}|S(x) / E(x)|^{2}\left(1+x^{2}\right)^{-1} d x<\infty$.

Каждая сопутствующая функция $S(S(0) \neq 0)$ порождает в $\mathscr{H}(E)$ оператор $B_{S}$, сопряженный к которому задается формулой

$$
\left(B_{S}^{*} f\right)(\lambda)=(f(\lambda) S(0)-S(\lambda) f(0)) / \lambda S(0), \quad f \in \mathscr{H}(E) .
$$

* Работа выполнена при поддержке Украинско-Израильского проекта научного сотрудничества (контракт № 2M/1516-97). 
При определенных условиях операторы $B_{S}$ являются модельными в классе всех вполне несамосопряженных операторов с двумерными мнимыми компонентами, характеристические матрицы-функции которых являются совершенными матрицами де Бранжа [4].

Если при некотором $\alpha \in \mathbb{R}$ функция $S_{\alpha}(\lambda):=e^{i \alpha \lambda}$ сопутствует пространству $\mathscr{H}(E)$, то $E$ есть функция ограниченного вида в области $\operatorname{Im} z>0$. Отсюда вытекает, что $E$ и все элементы из $\mathscr{H}(E)$ суть целые функции экспоненциального типа и, стало быть, пространству $\mathscr{H}(E)$ будут сопутствовать все функции $S_{\alpha}$, $-\omega \leqslant \alpha \leqslant \omega$, где $\omega:=h(E)$. Обозначим через $B_{\alpha}$ оператор, сопряженный к которому задается формулой $(2)$ с $S=S_{\alpha}$. Таким образом, мы получим семейство $B_{\alpha}(-\omega \leqslant \alpha \leqslant \omega)$ вольтерровых модельных операторов в пространстве де Бранжа $\mathscr{H}(E)$.

В этой статье важную роль играют пространства $\mathscr{H}(E)$, выделяемые следующим определением. Пространство де Бранжа $\mathscr{H}(E)$ назовем регулярнылм, если вес $|E(x)|^{2}$ удовлетворяет следующему условию на $\mathbb{R}[5]$ :

$$
\sup _{I}\left\{\frac{1}{|I|} \int_{I}|E(x)|^{2} d x \frac{1}{|I|} \int_{I}|E(x)|^{-2} d x\right\}<\infty,
$$

где $I$ - произвольный интервал, а $|I|$ - его длина. Нетривиальные примеры функций $E$, порождающих регулярные пространства $\mathscr{H}(E)$, можно найти в $[7]$, где они рассматривались по другому поводу. Условие регулярности пространства налагает ограничение на поведение функции $E$ вблизи $\mathbb{R}$.

Теорема 1. Пусть при некотором $\alpha \in \mathbb{R}$ функици $S_{\alpha}$ coпутствует регулярному пространству $\mathscr{H}(E)$. Тогда сущуествует полоса $0 \leqslant \operatorname{Im} z \leqslant \varepsilon$, свободная от корней функции $E^{*}$, и при каждом $\beta \in(0, \varepsilon]$

$$
0<\inf _{z \in \mathbb{R}+i \beta}\left|E^{*}(z) / E(z)\right| \leqslant \sup _{z \in \mathbb{R}+i \beta}\left|E^{*}(z) / E(z)\right|<1 \text {. }
$$

Судить о том, является ли пространство $\mathscr{H}(E)$ регулярным, можно по спектральной структуре модельных операторов $B_{S}$. В этой работе нас будут интересовать только вольтерровы операторы $B_{\alpha}$. Обозначим через $I_{\gamma}$ оператор интегрирования в $L_{2}(0, \gamma)$ :

$$
\left(I_{\gamma} h\right)(x)=i \int_{0}^{x} h(t) d t, \quad h \in L_{2}(0, \gamma) .
$$

Заметим, что операторы $I_{\gamma}$ диссипативны, т. е. $\left(I_{\gamma}-I_{\gamma}^{*}\right) /(2 i) \geqslant 0$.

ТеОРема 2. Пусть некоторая функция $S_{\alpha}$ conymствует пространству де Бранжа $\mathscr{H}(E)$. Если $\mathscr{H}(E)$ регулярно, то каждый оператор $B_{\alpha},-\omega \leqslant \alpha \leqslant \omega$, $\omega=h(E)$, подобен ортогональной сумме $I_{\omega+\alpha} \oplus\left(-I_{\omega-\alpha}\right)$ операторов интегрирования. Обратно, пусть существует полоса $0 \leqslant \operatorname{Im} z \leqslant \varepsilon$, свободная от корней функции $E^{*}$, и при некотором $\beta_{0} \in(0, \varepsilon]$ имеет место (3). Eсли некоторый оператор $B_{\alpha}$ подобен ортогональной сумме $B_{1} \oplus\left(-B_{2}\right)$, где $B_{i}-$ диссипативные операторы в сепарабельных гильбертовых пространствах $H_{i}$ $(i=1,2)$, то пространство $\mathscr{H}(E)$ регулярно.

Первая часть теоремы для регулярного пространства $\mathscr{H}(E)$ с $|E(x)|^{2} \asymp 1$, $x \in \mathbb{R}$, при дополнительном условии $\alpha \geqslant 0$ впервые доказана в [4]. Далее, из теоремы 2 вытекает, что в регулярных пространствах существуют операторы $B_{\alpha}^{-1}$ 
(неограниченные) и только при $\alpha=\omega$ полугруппа $\exp \left\{-i B_{\omega}^{-1} t\right\}, t \geqslant 0$, принадлежит классу $C_{0}$. Имеет место

ТЕОРема 3. Пусть некоторая функция $S_{\alpha}$ conутствует пространству $\mathscr{H}(E), \omega=h(E)$. Пространство $\mathscr{H}(E)$ регулярно тогда и только тогда, когда 1) существует полоса $0 \leqslant \operatorname{Im} z \leqslant \varepsilon$, в которой $E^{*}$ не обращаются $в$ нуль, и при некотором $\beta_{0} \in(0, \varepsilon]$ выполняется (3); 2) существует оператор $B_{\omega}^{-1}$ u полугруппа $\exp \left\{-i B_{\omega}^{-1} t\right\}, t \geqslant 0$, принадлежит классу $C_{0}$.

Аналогичное утверждение справедливо и для другого крайнего случая $\alpha=-\omega$.

2. Биортогональные разложения и интерполяция. Теперь к исследованию регулярных пространств де Бранжа можно применить результаты работ $[1,2]$. Во всех следующих ниже теоремах предполагается, что некоторая функция $S_{\alpha}$ conутствует пространству $\mathscr{H}(E)$, m. е. оператор $B_{\omega}, \omega=h(E)$, действует в $\mathscr{H}(E)$. Рассмотрим вектор-функцию

$$
G(z):=\left(I-z B_{\omega}\right)^{-1} g, \quad g \in \mathscr{H}(E),
$$

и зададимся вопросом: при каких $g$ из значений функции $G(z)$ можно составить безусловный базис пространства $\mathscr{H}(E)$ ?

Обозначим через $V$ множество аналитических в области $\operatorname{Im} z \leqslant 0$ функций $v$, допускающих внешне-внутреннюю факторизацию вида

$$
v(z)=s(z) w(z), \quad \inf _{z<0}\left\{|s(z)|+\left|e^{-2 i \omega z}\right|\right\}>0,
$$

где $s-$ внутренняя функция, а внешняя функция $w$ такова, что вес $|w(x-i 0)|^{2}$ удовлетворяет условию $\left(\mathrm{A}_{2}\right)$ на $\mathbb{R}$. Имеет место

ТеоремА 4. Для регулярных пространств де Бранжа следующие условия равносильнь:

1) существует такая последовательность $\Lambda:=\left\{\lambda_{k}\right\}_{-\infty}^{+\infty}, \inf _{k} \operatorname{Im} \lambda_{k}>-\infty$, ито семейство $\left\{G\left(\lambda_{k}\right)\right\}_{-\infty}^{+\infty}$ образует безусловный базис в $\mathscr{H}(E)$;

2) существуют такие константы $m, M(0<m<M<\infty)$, что для всех $h \in \mathscr{H}(E)$

$$
m\|h\|^{2} \leqslant \int_{\mathbb{R}}|\langle G(x), h\rangle|^{2}\|G(x)\|^{-2} d x \leqslant M\|h\|^{2} ;
$$

3) функция g допускает представление

$g(\lambda)=\lim _{\varepsilon \rightarrow+0} \frac{i}{\sqrt{2 \pi}} \int_{\mathbb{R}} k(\lambda, x) E^{-1}(x) B(x-i 0) e^{-i \delta x} P_{+}\left\{e^{2 i \omega x} v(x-i \varepsilon)(x-i \varepsilon)^{-1}\right\} d x$,

где ядро $k$ определяется равенством (1), B - произведение Бляшке в $\operatorname{Im} z<0$, составленное по корням функции $E, \delta:=\omega-\omega^{*}, \omega^{*}=h\left(E^{*}\right)$, и $P_{+}-$ортопроектор из $L_{2}(\mathbb{R})$ на класс Харди $H_{+}^{2}, v \in V$.

Отметим, что в формуле (5) предел и интеграл существуют в метрике пространства $\mathscr{H}(E)$. Теперь описание интересующих нас базисов дается следующей теоремой.

Теорема 5. Пусть $v=s w-$ внешне-внутренняя факторизация функции $v \in V$ и $G$-отвечающая $v$ в силу формул (4), (5) вектор-функция. Семейство $\left\{G\left(\lambda_{k}\right): \lambda_{k} \in \Lambda\right\}$, такое, что $\eta_{0}:=\inf _{k} \operatorname{Im} \lambda_{k}>-\infty$, образует безусловныии базис регулярного пространства $\mathscr{H}(E)$ тогда и только тогда, когда $\Lambda$ совпадает с множеством (простьх) корней целой функции ч экспоненциального 
типа, удовлетворяющей следующим условиям: 1) $\left.h(\varphi)=h\left(\varphi^{*}\right)=\omega ; 2\right)$ вес $|w(z)|^{-2}|\varphi(z)|^{2}$ удовлетворяет условию $\left(A_{2}\right)$ на какой-нибудь прямой $\mathbb{R}+i \eta$, $\left.\eta<\min \left\{0, \eta_{0}\right\} ; 3\right)$ последовательность $\left\{\lambda_{k}-i \eta: \lambda_{k} \in \Lambda\right\}$ отделима в смысле Карлесона, т.е.

$$
\inf _{n} \prod_{k \neq n} \frac{\left|\mu_{n}-\mu_{k}\right|}{\left|\mu_{n}-\bar{\mu}_{k}\right|}>0, \quad \mu_{k}:=\lambda_{k}-i \eta
$$

Если $|E(x)|^{2}$ удовлетворяет условию $\left(\mathrm{A}_{2}\right)$, то внешняя функция $v(z):=$ $E(z) B^{-1}(z) e^{-i \omega^{*} z}$ принадлежит классу $V$. Если считать, что $E(0) \in \mathbb{R}$ и, стало быть, $b(0)=0$, то такой функции $v$ по формуле $(5)$ отвечает $g(\lambda)=b(\lambda) /(\pi \lambda)$ и формула (4) приобретает вид

$$
\left(I-z B_{\omega}\right)^{-1}(b(\lambda) /(\pi \lambda))=e^{i \omega z} k(\lambda, \bar{z}) .
$$

Поэтому из теоремы 4 вытекает, что в регулярных пространствах $\mathscr{H}(E)$ существуют базисы из значений воспроизводящего ядра, описание которых дается теоремой 5. Сформулируем соответствующий результат в виде отдельной теоремы, ограничившись для простоты, случаем, когда последовательность $\Lambda$ расположена в верхней полуплоскости.

Теорема 6. Семейство $\left\{k\left(\lambda, \bar{\lambda}_{n}\right): \lambda_{n} \in \Lambda\right\}, \inf _{k} \operatorname{Im} \lambda_{k}>0$, образует безусловный базис регулярного пространства де Бранжа $\mathscr{H}(E)$ тогда и только тогда, когда $\Lambda$ совпадает с множеством (простых) корней некоторой сопутствующей этому пространству функции $S$, которая удовлетворяет следующим условиям: 1) $\left.h(S)=h\left(S^{*}\right)=\omega ; 2\right)$ вес $|E(x)|^{-2}|S(x)|^{2}$ удовлетворяет условию $\left(\mathrm{A}_{2}\right)$ на $\left.\mathbb{R} ; 3\right) \Lambda$ отделима в смысле Карлесона.

Если в этой системе положить $E(z)=e^{-i \omega z}, \omega>0$, и с помощью преобразования Фурье перейти к пространству $L_{2}(-\omega, \omega)$, то получим теорему Б. С. Павлова [6] об описании базисов из экспонент.

Системы, биортогональные к базисам из теоремы 6 , состоят из функций

$$
\frac{S^{*}(\lambda)}{\left(\lambda-\bar{\lambda}_{n}\right) \overline{S^{\prime}\left(\lambda_{n}\right)}} \quad\left(\lambda_{n} \in \Lambda\right) \text {. }
$$

Отсюда выводится, что каждая функция $f \in \mathscr{H}(E)$ допускает биортогональное разложение

$$
f(\lambda)=\sum_{\lambda_{k} \in \Lambda} \frac{c_{k} S(\lambda)}{\left(\lambda-\lambda_{k}\right) S^{\prime}\left(\lambda_{k}\right)}, \quad \int_{\mathbb{R}} \frac{|f(x)|^{2}}{|E(x)|^{2}} d x \asymp \sum_{\lambda_{k} \in \Lambda} \frac{\left|c_{k}\right|^{2} \operatorname{Im} \lambda_{k} e^{-2 \omega \operatorname{Im} \lambda_{k}}}{\left|E\left(\lambda_{k}\right)\right|^{2}},
$$

причем $c_{k}=f\left(\lambda_{k}\right), \lambda_{k} \in \Lambda$. Эти формулы подсказывают решение задачи интерполяции функциями из регулярных пространств де Бранжа.

Пусть $\Lambda=\left\{\lambda_{k}\right\}_{-\infty}^{+\infty}-$ произвольная последовательность комплексных чисел. Задачу об интерполяции в пространствах $\mathscr{H}(E)$ сформулируем так: какими должны быть $\Lambda$ и весовая последовательность $\left\{b_{k}\right\}_{-\infty}^{+\infty}$, чтобы интерполяционный оператор

$$
\mathscr{J}_{\Lambda} f:=\left\{f\left(\lambda_{k}\right)\right\}_{-\infty}^{+\infty}, \quad f \in \mathscr{H}(E)
$$


взаимно однозначно (и, значит, взаимно непрерывно) отображал $\mathscr{H}(E)$ на пространство $l_{2}\left\{b_{k}\right\}$ последовательностей $c:=\left\{c_{k}\right\}_{-\infty}^{+\infty}$ с нормой

$$
\|c\|^{2}=\sum_{-\infty}^{+\infty}\left|c_{k}\right|^{2} b_{k}<\infty .
$$

Последовательности $\Lambda$, описание которых дается теоремой 6 , назовем интерполяционными. Имеет место

Теорема 7. Если некоторая последовательность $\Lambda=\left\{\lambda_{k}\right\}_{-\infty}^{+\infty}, \inf _{k} \operatorname{Im} \lambda_{k}>0$, порождает взаимно однозначное отображение $\mathscr{J}_{\Lambda}$ регулярного пространства $\mathscr{H}(E)$ на пространство $l_{2}\left\{b_{k}\right\}$, то верны двусторонние оценки

$$
b_{k} \asymp \operatorname{Im} \lambda_{k} e^{-2 \omega \operatorname{Im} \lambda_{k}}\left|E\left(\lambda_{k}\right)\right|^{-2}, \quad k \in \mathbb{Z} .
$$

Далее, оператор $\mathscr{J}_{\Lambda}$ является изоморфизмом регулярного пространства $\mathscr{H}(E)$ на $l_{2}\left\{b_{k}\right\}$ с весом, удовлетворяющим условию (7), тогда и только тогда, когда последовательность $\Lambda$ интерполяционная. Единственное решение интерполяционной задачи $f\left(\lambda_{k}\right)=c_{k}\left(\lambda_{k} \in \Lambda\right)$ дается рядом (6), который сходится как по норме пространства $\mathscr{H}(E)$, так и равномерно на компактах плоскости.

Выражаю глубокую признательность Д. 3. Арову за многочисленные обсуждения результатов статьи.

\title{
ЛИТЕРАТУРА
}

1. Губреев Г. М. Функц. анализ и его прил., 33, вып. 1, $62-65$ (1999). 2. Губреев Г. М. Матем. сб́., 190, № 12 (1999). 3. De-Branges L. Hilbert spaces of entire functions. Prentice Hall, 1968. 4. Сахнович Л. А. Алгебра и анализ, 5, вып. 1. 3-80 (1993). 5. Гарнетт Дж. Ограниченные аналитические функции. Мир, М., 1984. 6. Павлов Б. С. ДАН СССР, 247, № 1, 37-40 (1979). 7. Джрбашян М. М. Изв. АН АрмССР, Математика, 19, № 2 (1984).

Южноукраинский педагогический госуниверситет, г. Одесса

Поступило в редакцию 9 декабря 1999 г.

УДК 517.938

\section{Проблема Гильберта-Арнольда и оценка цикличности полициклов на плоскости и в пространстве}

\author{
(c) 2001. В. Ю. КАлошин
}

1. Полициклы на плоскости. Мотивировкой для следующего определения является проблема Гильберта-Арнольда о равномерной ограниченности числа предельных циклов для типичных семейств гладких векторных полей на сфере $S^{2}$ (см., например, $[1,6]$ ).

ОПРЕДЕЛЕНИЕ 1. Полицикл $\gamma$ векторного поля $\dot{x}=v\left(x, \varepsilon^{*}\right), x \in S^{2}, \varepsilon^{*} \in B,-$ это циклически упорядоченный набор положений равновесия $p_{1}, \ldots, p_{k}$ (возможно, с повторениями) и набор фазовых кривых $\gamma_{1}, \ldots, \gamma_{k}$ (без повторений), их 